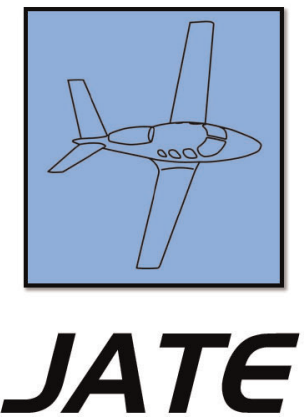

Journal of Aviation Technology and Engineering 9:2 (2020) 45-56

\title{
Airplane Pitch Response to Rapid Configuration Change: Flight Test and Safety Assessment
}

\author{
Ralph D. Kimberlin ${ }^{1}$, Markus Wilde ${ }^{2}$, Brian A. Kish ${ }^{3}$, and Isaac Silver ${ }^{4}$ \\ Florida Institute of Technology, Melbourne, FL 32901, USA
}

\begin{abstract}
This paper examines airplane response to rapid flap extension on seven general aviation airplanes. The scenario involves a pilot flying in the traffic pattern becoming distracted, abruptly extending flaps while looking outside the airplane, and failing to notice airspeed and pitch-attitude changes. The airplanes tested reached pitch forces of up to $36 \mathrm{lbf}$, meeting FAA requirements but exceeding the capability of $55 \%$ of the population. Flight data showed a pitch-up to more than $30^{\circ}$ in $5 \mathrm{~s}$ after flap extension, causing airspeed to drop below stall speed for four of the airplanes. At traffic pattern altitudes, stalling an airplane can be fatal. The NTSB lists over 1000 accidents caused by loss of control in the traffic pattern between 1982 and 2017. As general aviation airplanes do not carry flight data recorders, it is unknown how many of those accidents may have involved stalls caused by uncommanded response after flap extension. From the data gathered in flight, it seems possible some were. To improve safety, flight training should prepare students to anticipate rapid pitch changes during flap extension and retraction. In addition, airplane developers could interconnect flaps with the elevator, reduce horizontal tail size, or use a T-tail. The FAA should consider reducing the maximum pitch stick and wheel forces in 14 CFR §23.143 to $20 \mathrm{lbf}$ or less.
\end{abstract}

Keywords: loss of control, stall precursors, configuration change, flap-edge vortices

\section{Nomenclature}

$\mathrm{CFR}=$ Code of Federal Regulations

$\mathrm{CG}=$ Center of gravity

DOD $=$ Department of Defense

EASA $=$ European Union Aviation Safety Agency

$\mathrm{FAA}=$ Federal Aviation Administration

IMC $=$ Instrument meteorological condition

\footnotetext{
${ }^{1}$ Professor, Aerospace, Physics and Space Sciences.

${ }^{2}$ Associate Professor, Aerospace, Physics and Space Sciences.

${ }^{3}$ Associate Professor, Aerospace, Physics and Space Sciences.

${ }^{4}$ Chief Scientist, Energy Management Aerospace, LLC.
} 


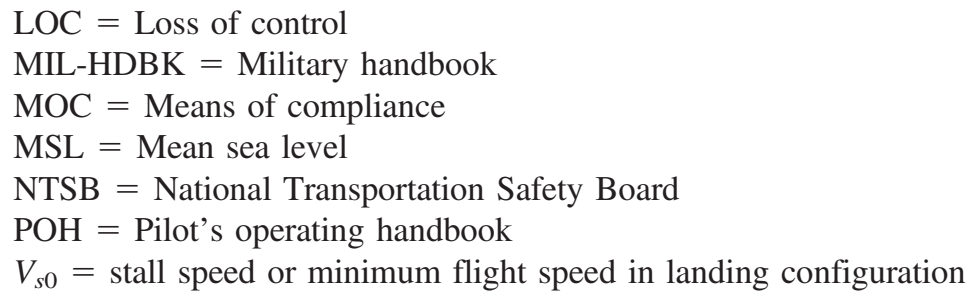

\section{Introduction}

Aviation safety must be considered one of the greatest engineering accomplishments of the past 100 years. For 2018, the National Transportation Safety Board (NTSB) reports a total of 38,515 U.S. transportation fatalities, of which only 393 were incurred in aviation (NTSB, 2018). Compared with air carrier operations, however, general aviation (GA) with 330 fatalities has a disproportionate accident rate. The NTSB publishes detailed annual aviation safety reviews (NTSB, 2020). In the period 2012-2017, the NTSB recorded 7630 accidents in GA, of which $18 \%$ (1396) resulted in fatalities. About $63 \%$ of the GA accidents occurred with fixed-wing aircraft flown in personal use. The defining event for about $45 \%$ of fatal accidents is inflight loss of control (LOC), defined by the NTSB as "loss of aircraft control while in flight, or extreme deviation from intended flightpath" (NTSB, 2011). In other words, a pilot either is distracted from the piloting tasks or is not paying sufficient attention to the airplane, leading to a significant loss in airspeed and/or altitude, ultimately resulting in a crash. To counter the high incidence of LOC accidents, the focus of past and current GA safety research has been on improving pilot awareness for energy, airspeed, and angle of attack through information and warning systems, designing airplanes for benign stall characteristics, and designing airplanes for benign post-stall spin characteristics. However, a detailed look at the published NTSB accident data shows that only $26 \%$ of all fatal accidents occur "en route," so at or around cruising altitude, whereas $35 \%$ occur during initial climb and approach, below $1000 \mathrm{ft}$ in altitude. If a stall or spin starts at such low altitude, the pilot does not have enough altitude/time to recover the airplane and convert altitude into airspeed before ground impact. This is highlighted by research conducted by the European Union Aviation Safety Agency (EASA). In 2008, the EASA analyzed 57 stall/spin accidents and determined their exact locations; 10 of the accidents occurred in the period 1999-2008 and involved airplanes designed to be "spin resistant," namely Cirrus SR-20 and SR-22; the rest were fatal fixed-wing GA accidents occurring in 2006. The analysis found that $79 \%$ of the accidents happened at altitudes below $1000 \mathrm{ft}$ and $84 \%$ of those low-altitude accidents occurred within the traffic pattern (Hankers et al., 2009). The available data clearly show that focusing on stall and spin qualities of airplanes is too late in the mishap chain to prevent $35 \%$ of the total fatalities in fixed-wing, personal use GA. In the USA alone, this represents on average 73 fatalities per year, or one every five days.

Therefore, additional research and development must be devoted to identifying and mitigating factors contributing to setting a pilot up to lose control of an airplane, resulting in a stall or spin. Some countermeasures are common sense, such as reducing distractions during the flight, heeding the "sterile cockpit" rule, and improving the accessibility of airspeed, altitude, and energy information through glass cockpits or heads-up displays. However, these countermeasures only address those pilots that pay attention to the piloting task and are fully aware of the cockpit environment. More critical are factors that can surprise inexperienced or distracted pilots during the most accident-prone phases of flight, in particular the final approach and the upwind leg during takeoffs and go-arounds. It is the experience of the test pilots involved in this study, gained over more than 15,000 cumulative flight hours accrued over 100 years of cumulative flight experience in aircraft of all types, that the airplane configuration changes during these flight phases, i.e. extending and retracting trailing edge flaps, can be a major contributor to the mishap chain leading to fatal accidents. The extension of flaps causes the shedding of flap-edge vortices which, under the wrong geometric conditions, can significantly alter downwash and dynamic pressure at the horizontal tail, changing the forces generated by the horizontal tail and thus the pitching moment of the airplane (Kimberlin, 2003). This can lead to an abrupt increase in longitudinal control forces and, if not compensated by the pilot, to rapid changes in pitch attitude, potentially setting the airplane up for a stall and ground impact.

Normal category ("general aviation") airplanes with less than 19 passengers and 19,000 $\mathrm{lb}$ maximum takeoff weight are regulated by the Code of Federal Regulations (CFR), Title 14, Part 23. Prescriptive control force limits were effectively removed from regulatory language with a 2017 rewrite of Part 23 (Amendment 64) with deference to a yetto-be-determined industry consensus standard. Therefore, the controllability requirement stated in 14 CFR \$23.2135 is that airplanes must be controllable and maneuverable during all phases of flight within the operating envelope and during configuration changes without exceptional piloting skill, alertness or strength (14 CFR §23.2135, 2017). 
Up until the issuance of Part 23 Amendment 64, 14 CFR §23.143(c) actually prescribed the maximum allowable control forces: For temporary application, the maximum pitch control force on a control stick was specified as $60 \mathrm{lbf}$ and the maximum pitch control force with one hand on a control wheel was specified as $50 \mathrm{lbf}$ (14CFR §23.14, 2012).

The maximum force limits specified in 14 CFR §23.143(c) have long been considered too high by the flight test and flying qualities community. A Federal Aviation Administration (FAA) study published in 2019 found that $68 \%$ of a representative sample of the current pilot population (male and female between 18 and 69 years of age) could not meet the $\$ 23.143(\mathrm{c})$ single-handed wheel (yoke) pitch requirement, and $80 \%$ could not meet the stick pitch force requirement (Beringer, 2019). The Department of Defense (DOD) guidelines for flying qualities of piloted aircraft, published in military handbook MIL-HDBK-1797, actually limit the maximum control forces due to pitch trim changes caused by the extension of secondary control devices such as flaps to $20 \mathrm{lbf}$ for a wheel and $10 \mathrm{lbf}$ for center-sticks (DOD, 1997).

Therefore, even when the rapid buildup of longitudinal control forces during flap extension stays within the 14 CFR \$23.143(c) limits, they can prove to be overwhelming for the majority of the pilot population and lead to lowlevel stalls and inflight LOC. Research into the interaction of transient flap-edge vortices and horizontal stabilizers is thus needed, in order to quantify the problem and to develop airplane design and flight training countermeasures.

The aviation safety aspects of flap-edge vortices have been studied quite exhaustively for transport category aircraft as defined in 14 CFR Part 25. However, these studies have almost exclusively focused on the contributions of the flap-edge vortices to the overall wake vortex during takeoff and landing (Breitsamter, 2011; Gerz et al., 2002; Özger et al., 2001; Rossow, 1999). For GA airplanes, literature research does not produce any detailed tests, simulations, or analyses focusing on the transient wake-tail interactions due to flap configuration changes. The purpose of this paper is to stimulate research in this critical area by demonstrating the severity of the configuration change response problem through flight test data for seven single-engine GA aircraft covering all typical wing-tail configurations; by proposing a suitable test methodology for manufacturers and regulators; by providing a phenomenological explanation of the configuration change effects based on airplane geometry; and by proposing a way for including the trim control forces and free airplane response in airworthiness certification methods. It is the intent of the authors that the data provided in this paper will lead to increased efforts in modeling and simulation of the response of small airplanes to configuration changes, serve the validation of such simulations, and ultimately change the way small airplanes are designed in order to minimize the configuration change effects.
The ultimate result of these efforts will be enhanced GA safety and a reduction in the number of GA fatalities.

The paper is organized as follows. Section II describes characteristics of the airplanes tested, and the flight test methods and procedures required to reproduce the work; section III reports the results of the flight test campaign; section IV provides the phenomenological analysis of the data; and section $\mathrm{V}$ discusses proposed countermeasures. Section VI concludes the paper.

\section{Materials and Methods}

\section{Test Aircraft}

Trim changes are fundamentally caused by changes in downwash at the horizontal tail due to configuration changes such as flap extension and retraction. Typically, airplanes with conventional tails undergo large changes in downwash and dynamic pressure at the tail with changes in power and flap position, and hence large trim changes (Kimberlin, 2003). To study the severity of airplane pitch response and pitch control force change during flap configuration changes and to evaluate the impact on flight safety, seven single-engine GA airplanes were subjected to a series of flight tests. The relevant characteristics of the airplanes are reported in Table 1 .

The airplanes evaluated represent some of the most popular and most typical types of GA airplanes, with conventional tails and T-tails, high wings and low wings, aluminum and composite construction. Except for the Mooney M20C, all airplanes have fixed landing gear. For all airplanes, full flaps is the standard position for landing. Six of the airplanes were certified by the FAA, one by the EASA, and all seven have been in service for more than a decade. The specific airplanes were selected because they were readily available as part of Florida Tech's aviation fleet or from local flight schools, not because any point was to be made regarding any model or manufacturer.

\section{Test Objectives and Procedures}

The test program had two objectives. The first test objective was to measure the pitch control forces required to maintain airspeed after a flap change. The second test objective was to measure airplane response with no pitch control inputs after a flap change. To increase test safety, the first test objective was always accomplished prior to the second test objective. This safety "buildup" enabled predictions of which configuration change would cause the most airplane response. Both test objectives were accomplished at nominal centers of gravity (CG) at near maximum gross weight. As the focus was on the effects of flap extension, power was kept constant during the experiments. In the absence of updated regulatory guidance, the flight tests followed the guidance in 14 CFR \$23.143 and 14 CFR 
Table 1

Airplane specifications (Cessna, 1978; Cirrus Aircraft, 2003; Diamong Aircraft, 2000; Mooney Aircraft Corporation, 1974; Piper Aircraft Corporation, 1968, 1979, 1994).

\begin{tabular}{|c|c|c|c|c|c|c|c|c|}
\hline Airplane & $\begin{array}{l}\text { Max. takeoff } \\
\text { weight (lb) }\end{array}$ & $\begin{array}{l}\text { Length } \\
\text { (ft) }\end{array}$ & $\begin{array}{c}\text { Wing span } \\
\text { (ft) }\end{array}$ & $\begin{array}{l}\text { Wing area } \\
\left(\mathrm{ft}^{2}\right)\end{array}$ & $\begin{array}{l}\text { Tail span } \\
\text { (ft) }\end{array}$ & $\begin{array}{l}\text { Aspect } \\
\text { ratio }\end{array}$ & $\begin{array}{c}\text { Wing } \\
\text { loading } \\
\left(\mathrm{lb} / \mathrm{ft}^{2}\right)\end{array}$ & $\begin{array}{c}\text { Power } \\
\text { loading } \\
(\mathrm{lb} / \mathrm{hp})\end{array}$ \\
\hline Piper PA-28-181 & 2550 & 24 & 35.5 & 170 & 12.9 & 7.41 & 15.0 & 14.2 \\
\hline Piper PA-32 & 3400 & 27.7 & 32.8 & 174.5 & 12.9 & 6.17 & 19.5 & 13.1 \\
\hline Cirrus SR20 & 3000 & 26 & 35.5 & 135.2 & 12.9 & 9.21 & 22.2 & 15.0 \\
\hline Diamond DA40 & 2535 & 26.3 & 39.2 & 145.7 & 10.9 & 10.53 & 17.4 & 14.1 \\
\hline Cessna C172N & 2300 & 26.9 & 36 & 174 & 11.3 & 7.45 & 13.2 & 14.4 \\
\hline Mooney M20C & 2575 & 23.2 & 35 & 170.5 & 11.8 & 7.18 & 15.1 & 14.3 \\
\hline Piper PA-28-180 & 2400 & 23.5 & 30 & 160 & 10.0 & 5.63 & 15.0 & 13.3 \\
\hline
\end{tabular}

\$23.145, measuring the single-hand pitch control force required to reestablish trim airspeed as flaps were extended to various positions (14 CFR §23.143, 2012; 14 CFR $\S 23.145,2012)$. The maneuvers were repeated with no pilot correction to reestablish trim airspeed. In other words, the airplane was allowed to respond freely to a change in flap setting. The collected data can be used to show what happens if the pilot is distracted and does not apply any corrections.

\section{Instrumentation}

For the first test objective, a hand-held force gauge inserted between the palm of the pilot's left hand and the control wheel or stick was used to measure pitch control forces. Simulating the downwind leg of the traffic pattern, the airplane was trimmed with $0^{\circ}$ of flaps at normal approach airspeed and power setting. The altitude band for testing was 2000 to $5000 \mathrm{ft}$ mean sea level (MSL). The pilots' only task was to maintain airspeed. Data were only gathered in smooth air. Once the pilots extended flaps, they recorded the pitch control force required to maintain airspeed.

For the second test objective, two cameras were installed in the cabin. One camera looked over the shoulder of the pilot to record the values on the cockpit instruments. The second camera looked over the right or left wing to get direct images of the pitch angles. The test procedure was as follows. Simulating the downwind leg of the traffic pattern, the airplane was trimmed with $0^{\circ}$ of flaps at normal approach airspeed and power setting. The altitude band for testing was 2000 to $5000 \mathrm{ft}$ MSL. Air quality was smooth. The pilots then abruptly extended flaps to full down and let the airplane respond with no further pitch control inputs. The pilots were allowed to apply small roll inputs to maintain wings-level flight, but no pitch controls were permitted. The pilots terminated the maneuver when they felt the airplane was about to stall. This means that the recorded free pitch response may in some cases not reflect the complete magnitude possible.

\section{Results}

The test pilot and flight test engineer read the force values directly off the hand-held force gauge and noted the values on the flight cards. The data for the changes in airplane pitch attitude and airspeed were reduced post-flight, by analyzing video of the cockpit instruments. Partial sets of the data provided here have been presented at conferences (Kish et al., 2016, 2019).

\section{Objective 1: Longitudinal Control Forces}

The first test objective was met on all airplanes. Table 2 lists the static pitch control forces required to maintain altitude after the final flap setting was achieved. As is typical for flight tests, the number of available data points is small and the data reported represent the consensus of the three test pilots involved after executing the test point multiple times. They account for variations in pilot technique along with measurement uncertainties. As expected for airplanes certified by the FAA, all of the forces were less than the $50 \mathrm{lbf}$ maximum required by 14 CFR $\$ 23.143(\mathrm{c})$.

The forces measured for the M20C, DA40, and PA-28180 were negligible. This suggests that extending the flaps into any setting will be uneventful. A pilot could even fly the airplane for a long time at those forces without re-trimming. These pitch force results were a substantial contrast compared to the results for the PA-32, PA-28-181, and $\mathrm{C} 172 \mathrm{~N}$. The required $35 \mathrm{lbf}$ of push force must be considered

Table 2

Longitudinal control force after full flap extension.

\begin{tabular}{lc}
\hline Airplane & Force (lbf) \\
\hline PA-32 & 36 push \\
PA-28-181 & 35 push \\
C172N & 35 push \\
SR20 & 22 push \\
M20C & 5 pull \\
DA40 & 4 push \\
PA-28-180 & 2 push \\
\hline
\end{tabular}



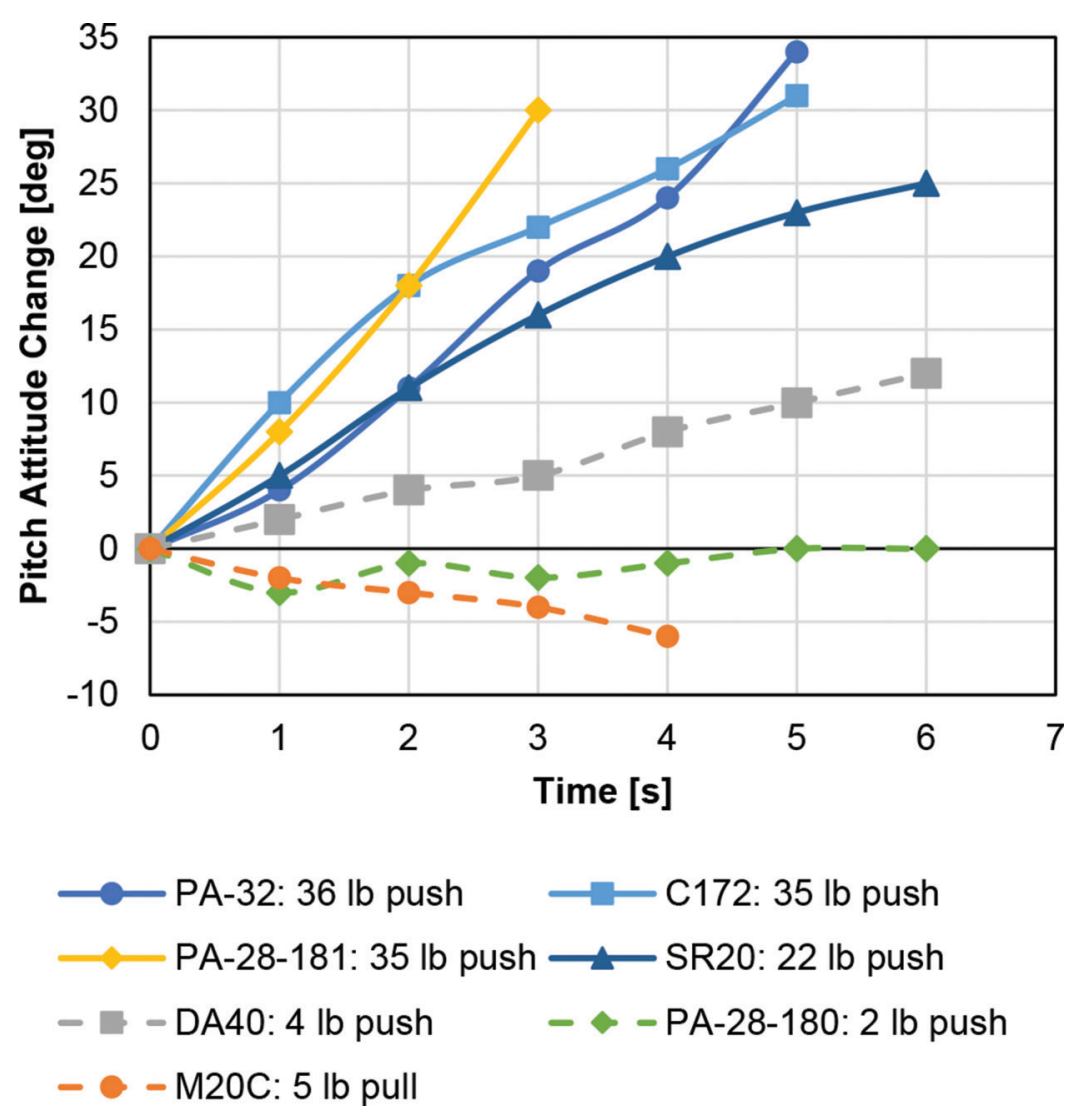

Figure 1. Pitch attitude versus time.

dangerous, as recent FAA research has shown that $55 \%$ of the population are not able to meet this force level for lefthanded yoke push (Beringer, 2019). Holding those forces without re-trimming would also not pass maximum force requirement for prolonged application specified in $14 \mathrm{CFR}$ \$23.143(c). Using the trim wheel to compensate for the additional control force is possible with forces achievable by all pilots. However, during one of the flights on the C172N, it took 9.5 turns of the trim wheel to balance the aircraft after arresting the pitch-up motion with the yoke. The experienced test pilot completed this in about $4 \mathrm{~s}$. As Figure 1 shows, the airplane would have pitched up by $25^{\circ}$ over this time.

\section{Objective 2: Free Pitch Response}

The second test objective was also met on all airplanes. Figure 1 shows pitch attitude versus time for the different pitch trim forces required to maintain altitude. For four of the seven airplanes, the change in pitch attitude is very rapid, with rates at $4-10 \%$. The free responses in Figure 1 represent what happens if the pilot does not apply the required force. As can be expected, the airplanes with the highest trim force changes have the highest pitching moment and thus the largest magnitude and highest rate in the free pitch response. In the PA-32, PA-28-181, C172N, and SR20, the pilot actually had to come on the controls to prevent the airplane from continuing to pitch up and stall.

Airspeed over time for the seven airplanes is reported in Figure 2. The airspeed is normalized over the stall speed in landing configuration, $V_{s 0}$. As is clearly evident, the $\mathrm{C} 172 \mathrm{~N}$ and PA-32 reached stalling conditions within 5-5.5 s. The PA-28-181 would have stalled within $4 \mathrm{~s}$, had the pilot not aborted the maneuver.

Figures 1 and 2 show how quickly an airplane can get to an unsafe condition during abrupt configuration changes if the pilot response is lagging. With control forces of up to $36 \mathrm{lbf}$ overpowering an unprepared pilot, the airplanes can reach stall attitude and speed within as little as $4 \mathrm{~s}$. If this happens in the traffic pattern, a fatal outcome is likely, as evident in the accident statistics. Therefore, the authors consider the airplane response of the PA-32, PA-28-181, C172N, and SR20 to abrupt flap extension from $0^{\circ}$ to $40^{\circ}$ to be unsafe. The remaining questions are: What could be the root causes and what can be done about mitigating the problem?

\section{Analysis}

The horizontal tail of an airplane is typically designed to meet requirements for longitudinal stability and control characteristics around common trim points. The pitching moment of an airplane is dominated by the contributions of the wing and of the horizontal tail, in terms of both their 

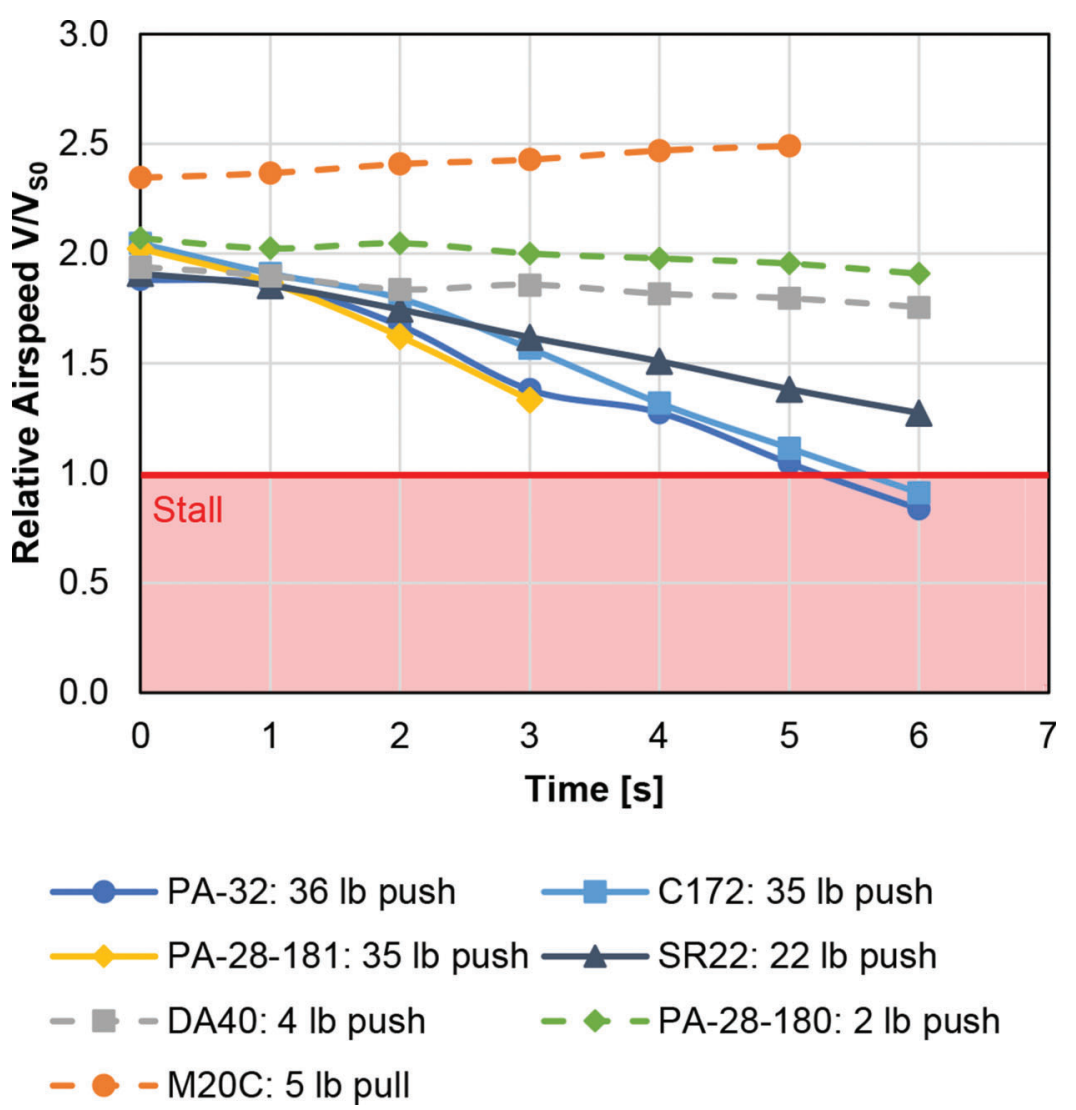

Figure 2. Airspeed versus time.

zero-lift pitching moments and the angle-of-attack-dependent pitching moments. The simple flap systems used on GA airplanes function by changing the camber of the airfoil, thus increasing the lift at zero angle of attack. At the same time, increasing the camber of an airfoil also generates a negative zero-lift moment. Therefore, extending the flaps should result in a nose-down pitching moment and an associated pull force. The significant nose-up moment observed in flight tests cannot be explained by wing aerodynamics, but only by the aerodynamic interaction of the wing and the horizontal tail. The interaction between the wing and the tail is typically accounted for by the downwash angle (Etkin \& Reid, 1995; Phillips et al., 2002; Silverstein \& Katzoff, 1939). The downwash at the tail is caused by the circulation around the wing, which is an inevitable byproduct of the generation of lift. As the horizontal tail is typically trimmed at negative angle of attack to produce a downward lift force and thus a nose-up pitching moment, any increase in downwash from the wing would generate a more downward force at the tail and thus an increased nose-up pitching moment. Therefore, an abrupt change in downwash angle with the extension of the flaps is the best candidate to explain the abrupt pitching response and rapid change in longitudinal control forces observed.

The downwash cannot be measured directly in flight tests without installation of a suite of dedicated instrumentation that would require the recertification of the airplane. Wind tunnel experiments using scaled models to accurately reproduce the aerodynamic conditions over the short length between wings and tail were outside the scope of the project presented in this paper. Computational fluid dynamics simulations require experimental validation from either flight tests or wind tunnel experiments. Therefore, the team decided to use analytical methods to determine whether the effects observed in flight tests can be explained by downwash-tail interaction, or whether more complex flap-edge vortex-tail interaction must be considered.

\section{Downwash-Tail Interaction}

The magnitude of the downwash can be estimated by using the lifting line theory (Phillips et al., 2002) or methods based on empirical data (Finck, 1978). Literature does not provide any closed-form analytical model that can be used to directly calculate the change in downwash angle due to a change in flap setting. However, the U.S. Air Force Stability and Control DATCOM provides a method based on empirical data (Finck, 1978, pp. 4.4.1-8). The method uses the change in lift coefficient due to flap extension, calculated from the change in stall speed given in the pilot's operating handbook (POH), and the height of the tail in relation to the wing span to estimate the change in downwash angle on the horizontal tail. Assuming that all other 


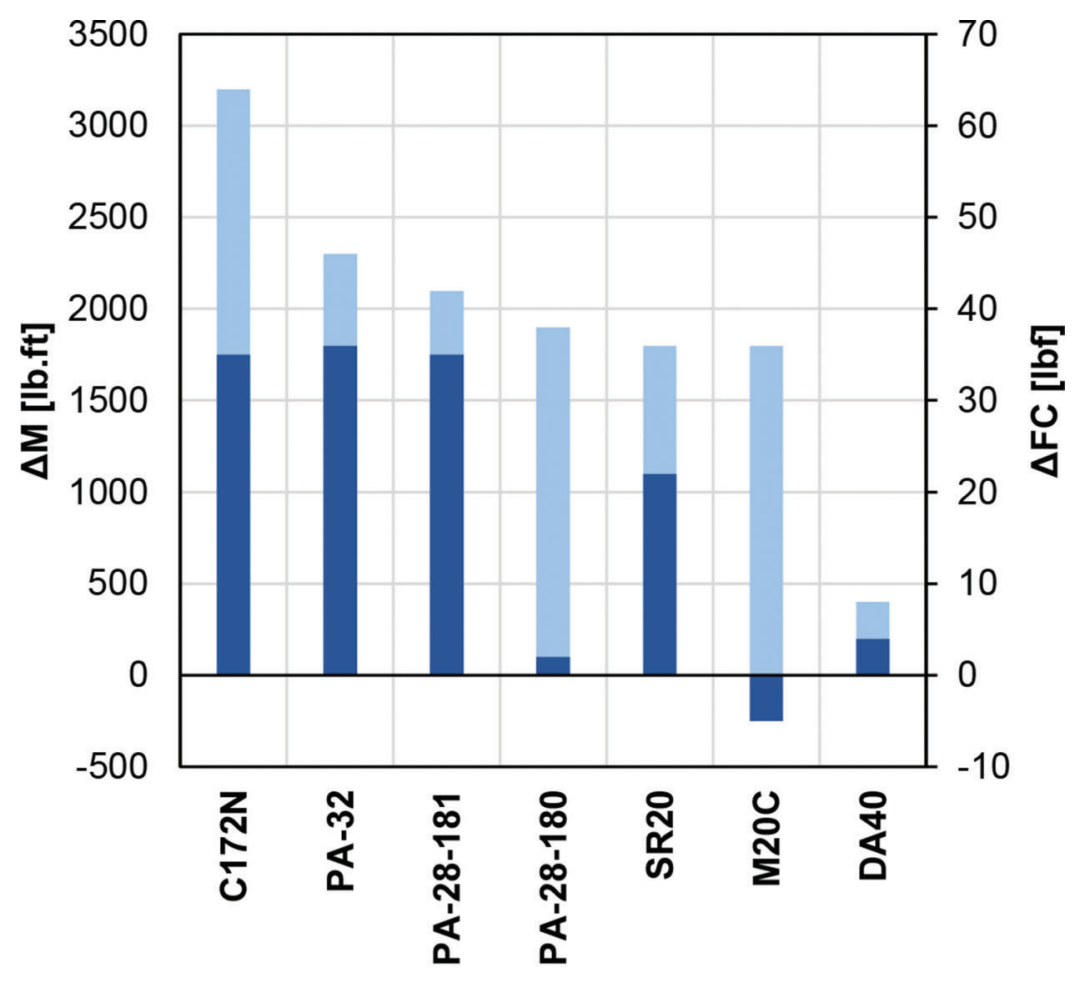

\section{Expected Pitching Moment Change from Downwash \\ - Measured Control Force Change}

Figure 3. Comparison of expected pitching moment change and measured control force change.

aerodynamic parameters remain unchanged during flap extension, the change in downwash angle directly drives the change in tail lift coefficient and the resulting change in airplane pitching moment. Figure 3 shows the control force changes measured versus the changes in pitching moment expected from the estimated changes in downwash angle due to flap extension.

As expected, the $\mathrm{C} 172 \mathrm{~N}$, with the tail below the wing, exhibits the highest change in downwash angle, whereas the DA40, with a T-tail, exhibits the lowest. The results also show almost identical changes in downwash angle for the PA-28-181 and PA-28-180. This also fits expectations, as the two airplanes are almost identical in configuration and very similar in dimensions and weight.

For aircraft with reversible, mechanical control systems such as typical GA airplanes, changes in pitching moment translate linearly into changes in longitudinal control force. With the PA-32 and PA-28-181 having almost identical control systems and tails of almost identical size, the fact that they should exhibit comparable pitching moments and control force changes meets expectations. The differences in moment-to-force ratios between PA-32/PA-28-181, C172N, and SR20 can potentially be explained by different nose-down, zero-lift moment of the cambered airfoil and by different gear ratios in the mechanical control linkages, but a detailed analysis of the control systems is beyond the scope of this paper. What can definitely not be explained using the downwash-tail interaction and control system gear ratios is the negative (pull) control force change for the $\mathrm{M} 20 \mathrm{C}$, and the large difference in control force change between the PA-28-180 and PA-28-181. As can be seen in Figure 5, the PA-28-181, PA-28-180, and PA-32 are almost identical airplanes, with the primary difference being the span, not the area, of the horizontal tail.

\section{Flap-Edge Vortex-Tail Interaction}

Vortices are typically thought of as a wingtip phenomenon. However, when flaps are extended, stable vortices form around both the inboard and outboard edges of the flap and flow off tailwards and inboard (Devenport et al., 1996; Lombardi \& Skinner, 2005; Schell et al., 2000; Shekarriz et al., 1993; Zuhal, 2001). For a number of span lengths, the wingtip vortices and flap-edge vortices are distinct vortex structures, with the flap-edge vortices being the strongest (Breitsamter et al., 2002; Greenblatt et al., 2009). The vortices then merge behind the airplane, becoming a major safety concern in commercial aviation (Crouch, 2005).

The span-wise location of a rolled-up tip vortex is not directly at the tip or outer edge of the lifting surface, but rather a certain distance inboard, depending on the aspect ratio and the taper ratio (Phillips et al., 2002). For certain combinations of flap location, flap span, and tail span, the 

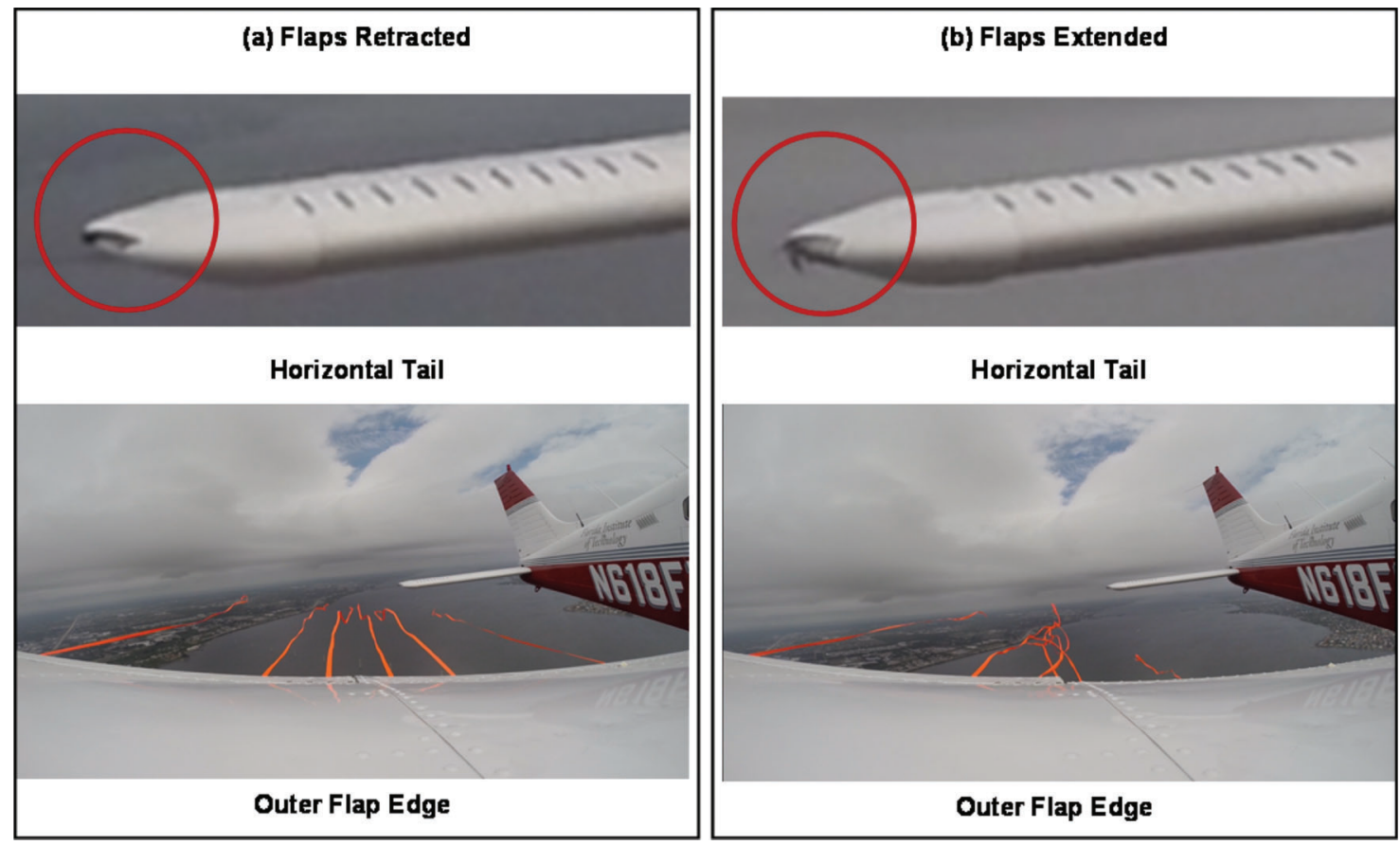

Figure 4. Flap-edge vortex and flap-tail interaction on PA-28-181.

flap-edge vortices may touch the horizontal tail. In that case, the outboard flap vortex, which is the dominating vortex in the wake of the wing (Breitsamter et al., 2002), would add significant downwash, whereas the inboard flap vortex would reduce the downwash at the tail. Flight tests demonstrate that significant flap-edge vortices form on GA airplanes during flap extension. Figure 4 shows photos taken on a PA-28-181, making visible the formation of the flap-edge vortex and the interaction with the horizontal tail. In Figure 4(a) the flaps are retracted. The streamers taped to the wing around the outer flap edge flow off straight and the tufts on the horizontal tail are pressed flat against the surface by the steady flow. Figure 4(b) shows the situation $4 \mathrm{~s}$ after flap extension. The streamers are twisted into the vortex flowing off the flap edge. The tufts are detached from the tail and are moving with the turbulence of the flapedge vortex interacting with the tail.

The PA-28-181, PA-28-180, and PA-32 are good test cases for the interaction between flap-edge vortex and horizontal tail. The PA-28-181 and PA-28-180 have flaps with identical area and span. The flaps start flush at the fuselage, thus assumedly preventing the formation of a defined inboard flap vortex. Figure 5(a) shows how an outer flap-edge vortex flowing off the flap at $85 \%$ span, combined with some additional inwards drift, would directly impact the tail of the PA-28-181, but not the tail of the PA-28-180, matching the flight test data shown in Figure 4 . The outer flap-edge vortex directly impinging on the horizontal tail would impart an additional downwash onto the tail, which may account for the significant difference in control force change between the two airplanes. In comparison, the PA-28-181 and PA-32 also have almost identical flap locations and spans, but also tails of identical span (see Figure 5(b)). Therefore, both tails would be impacted by the outboard flap-edge vortex, resulting in similar changes in control forces during flap extension.

If this hypothesis is correct, similar effects should be evident for the other airplanes of this study. The C172N (Figure 6(a)) has the inner edge of the flaps flush against the fuselage. The ratio between flap span and tail span seems conducive for interaction between the tail and the outboard flap-edge vortices. However, as the change in pitching moment expected from the conventional downwash analysis for the combination of high wing and conventional tail is almost twice that for the PA-32/PA-28180/PA-28-181, the flap-edge vortex-tail interaction may not be needed to explain the high longitudinal control force change measured. The flaps of the SR20 (Figure 6(b)) extend far enough outboard of the horizontal tail, so that an impact of the outboard flap-edge vortex on the tail is not expected. The inboard flap edge is sufficiently separated from the fuselage, so that a defined inboard flap vortex forms. The inboard flap vortex circulates in the opposite direction to the outboard flap tip. As the fuselage is nearby, the inboard flap vortex is expected to be weaker than the outboard flap vortex. Whether the interaction of the inboard flap vortex and the tail has any significant effect on the change in pitching moment and control force during flap extension cannot be answered based on the available data. The combination of low wing and T-tail in the DA40 (Figure 6(c)) leads to the downwash analysis resulting in a very low pitching moment. The outboard flap vortex passes well outboard and under the horizontal tail, so that no impact is expected. Therefore, the low control force change 


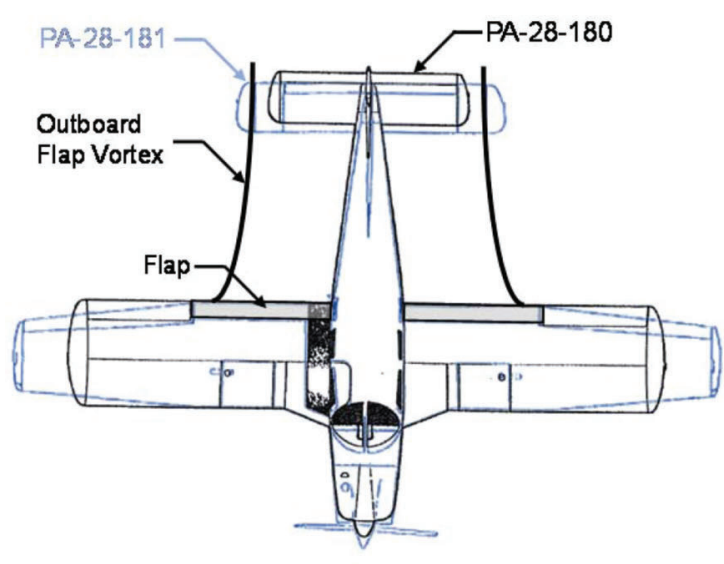

(a)

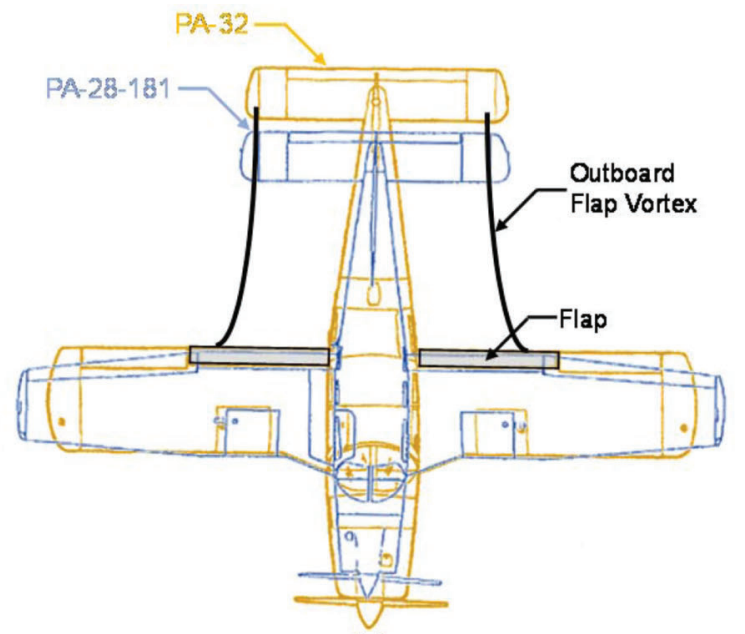

(b)

Figure 5. Airplane comparison and hypothesized flow of flap-edge vortex towards horizontal tail: (a) PA-28-181 versus PA-28-180; (b) PA-28-181 versus PA-32.

measured for the DA40 fits expectations. The nose-down pitching response and resultant negative control force change for the M20C (Figure 6(d)) can be attributed to three factors. First, the flap span of the M20C is substantially larger than for any of the other airplanes, spanning far beyond the tips of the horizontal tail, thus preventing effects from the flap-edge vortices. Second, although not a T-tail, the horizontal tail of the M20C is mounted higher up than on the PA-32, PA-28180, PA-28-181, and $\mathrm{C} 172 \mathrm{~N}$, thus reducing the downwash and nose-up moment. Third, based on the flight experience gained during the test campaign, the authors suspect that the airfoil used on the M20C generates a high nose-down, zerolift moment with flaps extended, thus overcoming the nosedown moment generated by the tail.

\section{Discussion}

The combination of flight test data and analytical modeling of the downwash changes during flap extension provides strong support for the hypothesis that the interaction of flap-edge vortices and the horizontal tail can cause unexpected, abrupt changes in airplane flight characteristics. As flaps are typically deployed and retracted at low altitude in the traffic pattern, the flap-edge vortex-tail interaction is assumed to be a significant contributor to LOC accidents. The scenario involving a pilot flying in the traffic pattern who becomes distracted, abruptly extends flaps while looking outside the airplane, and fails to notice any rapid changes in airspeed and pitch attitude is likely to occur. Another possible scenario involves a less experienced, single pilot in a high-workload, instrument meteorological conditions (IMC) approach. Many pilots execute configuration changes at critical points during such approach. Approach flaps may be selected upon intercepting the glideslope. Full flaps may be selected upon visually acquiring the runway at low altitude during the IMC approach.
The airplane free response should not add to the pilot workload problem. Ideally, a pilot should be able to extend the flaps and have the airplane remain in balance, with zero pitch rate. Addressing the issue of abrupt pitching motion after flap extension requires changes to pilot training and efforts by airplane manufacturers, supported and encouraged by the regulating authorities.

First, the potential for significant pitch response and changes in stick/wheel forces must be included in pilot training. Students must be trained to anticipate a rapid buildup of forces and to be ready to stop the pitching motion before the airplane attitude reaches critical limits. But this must be connected with an overall increased emphasis on a pilot's attention to the flying task and awareness of the energy state of the airplane. Although certainly not all LOC accidents are caused by the configuration change response, the majority of these accidents can be prevented by pilots having a better understanding of the energy reserves of the aircraft in relation to stall speed and above-ground altitude (Merkt, 2013). Changes to pilot training can be a highly effective and the most immediate measure to counter the configuration change problem.

Second, for new airplane designs, the effects of flap-edge vortex-tail interaction must be considered in tail sizing. This could result in favoring horizontal tails with shorter span or T-tails, and in interconnecting the flaps with the elevator. At the time of writing, no sufficient body of test data exists to adapt design software and parametric sizing methods. Therefore, the authors encourage civil aviation authorities and national aerospace research agencies to support efforts in numerical simulation, wind tunnel experimentation, and flight testing to close this knowledge gap. To give direction to designers of new airplanes, the maximum allowable temporary control force must be reduced. Based on their experience in multiple flight test campaigns, the authors recommend setting the maximum 


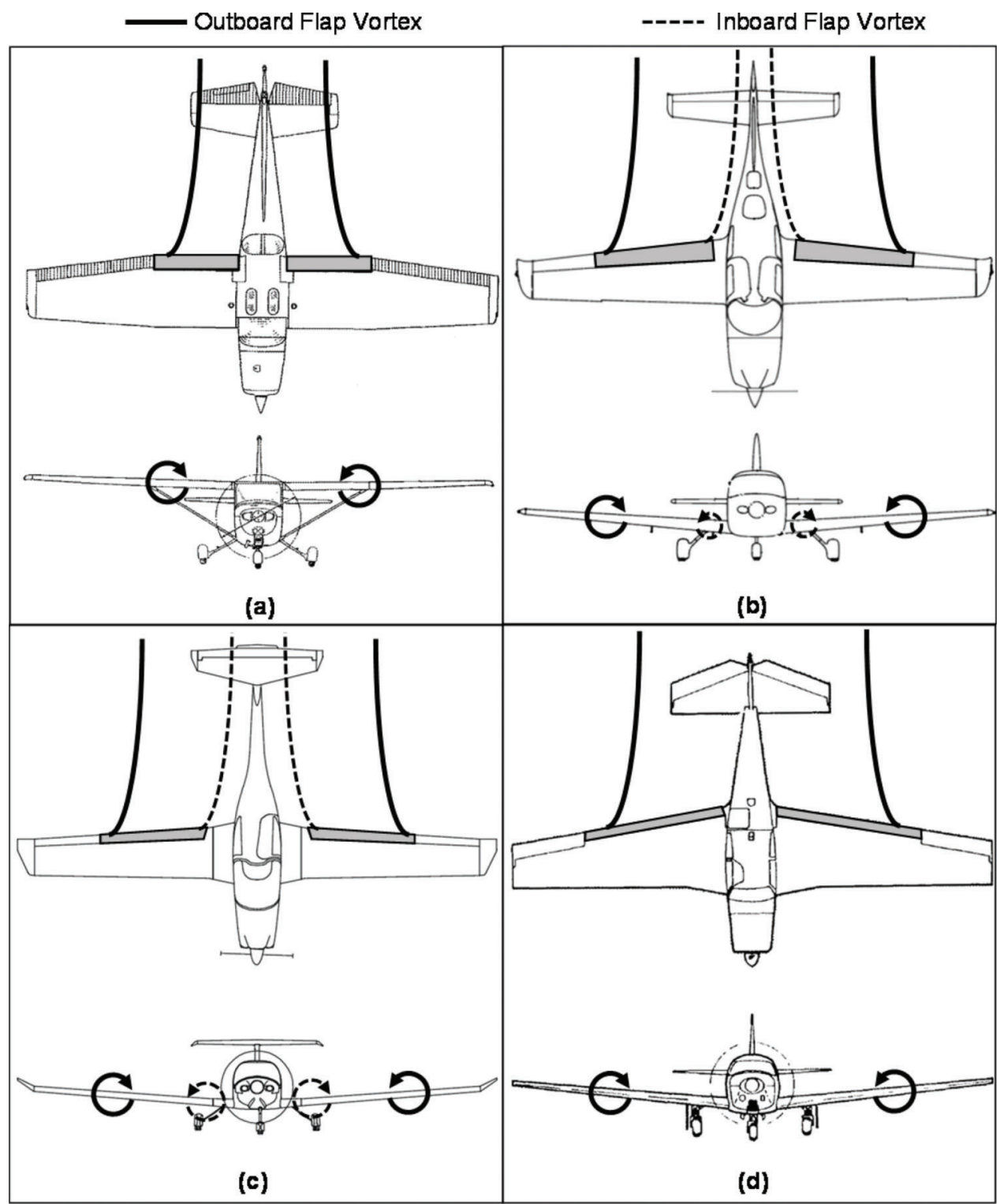

Figure 6. Hypothesized impact of flap-edge vortices on horizontal tails.

single-handed control force to $20 \mathrm{lbf}$, instead of the current 50 lbf.

Third, electromechanical measures can be taken to modify existing airplanes. If an airplane shows a direct and linear relationship between flap extension angle and control force change, interconnects between the flaps and elevator can reduce or eliminate the force change. A design change of this magnitude would require recertification by the authorities.

Fourth, the authors recommend that civil aviation authorities set the framework for changes in airplane design by amending the existing regulations and the associated means of compliance (MOC). With the introduction of performance-based regulations and MOC using airworthiness certification scores, such as the recently accepted ASTM standard specification for low-speed flight characteristics of airplanes (ASTM International, 2018), a portion of the available scores can be dedicated to the magnitude and direction of control force changes along with the free response angle and rates resulting from flap extension/retraction.

The combination of measures described can contribute significantly to reducing the number of fatal LOC accidents by moving the emphasis of pilot training, airplane design, and certification away from stalling and post-stall behavior to detrimental effects higher up in the mishap chain.

\section{Conclusion}

GA has an unacceptably high rate of fatal accidents, a substantial fraction of which can be attributed to inflight LOC. The majority of fatal LOC accidents occur in the traffic pattern around airfields, where the airplane is low and slow, leaving the pilot insufficient time to correct 
control errors. At the same time, the pilot cannot devote undivided attention to piloting, as configuration management, communication with air traffic control, and traffic observation consume significant fractions of attention bandwidth. In this critical phase of flight, any abrupt and unexpected change in the flying behavior of the aircraft can set up a pilot for a fatal accident. The flight tests documented in this paper, conducted with seven common and popular GA airplanes, show that the required longitudinal control force after rapid extension of flaps almost instantaneously reaches levels not achievable by the majority of the current pilot population. The tests also show that, if the pilot is not able to exert the control force required to stop the pitching motion of the airplane, the airplane's free pitching response results in rapid buildup of pitch angles and associated airspeed decrease, within seconds leading to stall conditions. Both control force change and free pitching response are caused by the change in airplane pitching moment due to flap extension, which is typically explained in terms of (1) additional nose-up moment due to the wing-induced downwash angle at the tail increasing with the wing lift coefficient and (2) an additional nose-down moment due to the camber of the wing increasing with flap extension. For the majority of the airplanes tested in this research, neither of the two contributions can explain the substantial nose-up change in pitching moment. Instead, the flight test data show strong indications that the direct interaction between the outer flap-edge vortices and the horizontal tail must be considered. If the horizontal tail is mounted at about the same height as the wings, and if the span of the flaps is similar to the span of the tail, the flap-edge vortex can impact the horizontal tail, adding substantial downwash and thus causing the large change in pitching moment, longitudinal control force, and nose-up pitching motion. In general, airplanes with T-tails and airplanes with high flap spans show the lowest configuration change effects. Based on the available data, (1) aircraft designers should consider this interaction when sizing flap systems and aircraft empennages, (2) regulators should include the configuration change response in airworthiness certification and decrease the maximum permissible longitudinal control force, and (3) after-market means for elevator-flap interconnects could be developed. In combination, these methods could eliminate abrupt airplane behavior during configuration change from the incident chain leading to LOC accidents. In addition, the authors also encourage the field to conduct further numerical and experimental research to better understand the phenomenon.

\section{Funding Sources}

The FAA supported this research through contract DTFACT-17-C-00001.

\section{Acknowledgments}

The team would like to thank the FAA for funding this effort and participating in the flight campaigns and analysis. David Sizoo and Ed Kolano flew as test pilots. David Webber and Ross Schaller flew as flight test engineers. Robert McGuire managed the program. The PA-28-180, PA-32, and PA-28-181 are part of the Florida Tech Flight Test Engineering fleet. The PA-28-180 is the personal airplane of co-author Ralph Kimberlin. Derek Fallon and C. J. Modine from Melbourne Flight Training provided the SR20 and DA40 and flew as pilots-incommand. The M20C was leased from the Matison family.

\section{References}

14 CFR $\$ 23.143$ (2012).

14 CFR $\$ 23.145$ (2012).

14 CFR §23.2135 (2017).

ASTM International, "Standard Specification for Low-Speed Flight Characteristics of Aircraft," ASTM F3180/F3180M-18, ASTM International, West Conshohocken, PA, 2018.

Beringer, D. R. (2019). NextGen Final Report: Data for Updating CFR Part 25.143 and Potential Reference Standards for Part 23, 27, and 29 Aircraft: An Evaluation of Muscular Force That can be Applied to Flight Controls. Federal Aviation Administration, Washington, DC.

Breitsamter, C. (2011). Wake vortex characteristics of transport aircraft. Progress in Aerospace Sciences, 47(2), 89-134.

Breitsamter, C., Bellastrada, C. \& Laschka, B. (2002). Investigations on the turbulent wake vortex flow of large transport aircraft. ICAS 2002 Congress.

Cessna. (1978). Pilot's operating handbook Skyhawk: Cessna Model $172 N$. Wichita, KS: Cessna Aircraft Company.

Cirrus Aircraft. (2003). Pilot's operating handbook and FAA approved airplane flight manual for the Cirrus Design SR20. Duluth, MN: Cirrus Design Corporation.

Crouch, J. (2005). Airplane trailing vortices and their control. Comptes Rendus Physique, 6, 487-499.

Department of Defense. (1997). Flying qualities of piloted aircraft. Department of Defense Handbook, MIL-HDBK-1797, Washington, DC.

Devenport, W. J., Rife, M. C., Liapis, S. I., \& Follin, G. J. (1996). The structure and development of a wing-tip vortex. Journal of Fluid Mechanics, 312, 67-106.

Diamong Aircraft. (2000). Airplane flight manual DA40. Wiener Neustadt, Austria: Diamond Aircraft Industries GmbH.

Etkin, B., \& Reid, L. D. (1995). Dynamics of flight: Stability and control (3rd ed.). New York, NY: Wiley.

Finck, R. D. (1978). USAF Stability and Control DATCOM. Air Force Wright Aeronautical Laboratories, AFWAL-TR-83-3048, Dayton, OH.

Gerz, T., Holzäpfel, F., \& Darraq, D. (2002). Commercial aircraft wake vortices. Progress in Aerospace Sciences, 38(3), 181-208.

Greenblatt, D., Vey, S., Paschereit, O. C., \& Meyer, R. (2009). Flap vortex management using active gurney flaps. AIAA Journal, 47(12), 28452856.

Hankers, R., Pätzold, F., Rausch, T., Kickert, R., Cremer, M. \& Troelsen, J. (2009). Safety aspects of light aircraft spin resistance concept. Cologne: European Aviation Safety Agency.

Kimberlin, R. D. (2003). Flight testing of fixed-wing aircraft. Reston, VA: AIAA Education Series. 
Kish, B. A., Bernard, T., \& Kimberlin, R. (2016). A limited investigation of airplane response to flap extension. IEEE Aerospace Conference, Big Sky, MT.

Kish, B. A., Wilde, M., Kimberlin, R., Silver, I., Sizoo, D. G., Webber, D., Kolano, E., Schaller, R., \& Toepfer, M. (2019). Trim forces and free response to configuration changes on general aviation aircraft. IEEE Aerospace Conference, Big Sky, MT.

Lombardi, G., \& Skinner, P. (2005). Wing-tip vortex in the near field: An experimental study. Journal of Aircraft, 42(5), 1366-1368.

Merkt, J. R. (2013). Flight energy management training: Promoting safety and efficiency. Journal of Aviation Technology and Engineering, 3(1).

Mooney Aircraft Corporation. (1974). Mooney Ranger operators manual: Model M20C. Kerrville, TX: Mooney Aircraft Corporation.

National Transportation Safety Board. (2011). Review of U.S. civil aviation accidents 2007-2009. Washington, DC: NTSB.

National Transportation Safety Board. (2018). US transportation fatalities in 2018-by mode. Retrieved December 10, 2020, from https://www. ntsb.gov/investigations/data/SiteAssets/Pages/Data_Stats/USTransportation-Fatalities-2018.pdf

National Transportation Safety Board. (2020). Aviation statistics-Annual summaries of US civil aviation accidents. Retrieved December 10, 2020, from https://www.ntsb.gov/investigations/data/Pages/aviation_stats.aspx

Özger, E., Schell, I., \& Jacob, D. (2001). On the structure and attenuation of an aircraft wake. Journal of Aircraft, 38(5), 878-887.
Phillips, W. F., Anderson, E. A., Jenkins, J. C., \& Sunouchi, S. (2002). Estimating the low-speed downwash angle on an aft tail. Journal of Aircraft, 39(4), 600-608.

Piper Aircraft Corporation. (1968). Cherokee Six owner's handbook. Vero Beach, FL: Piper Aircraft Corporation.

Piper Aircraft Corporation. (1979). Cherokee C owner's handbook. Vero Beach, FL: Piper Aircraft Corporation.

Piper Aircraft Corporation. (1994). Archer III pilot's operating handbook and FAA approved airplane flight manual. Vero Beach, FL: Piper Aircraft Corporation.

Rossow, V. J. (1999). Lift-generated vortex wakes of subsonic transport aircraft. Progress in Aerospace Sciences, 35(6), 507-660.

Schell, I., Özger, E., \& Jacob, D. (2000). Influence of different flap settings on the wake-vortex structure of a rectangular wing with flaps and means of alleviation with wing fins. Aerospace Science and Technology, 4(2), 79-90.

Shekarriz, A., Fu, T. C., Katz, J., \& Huang, T. T. (1993). Near-field behavior of a tip vortex. AIAA Journal, 31(1), 112-118.

Silverstein, A., \& Katzoff, S. (1939). Design charts for predicting downwash angles and wake characteristics behind plain and flapped wings, NACA-TR-648, Washington, DC.

Zuhal, L. R. (2001). Formation and near-field dynamics of a wing tip vortex [Doctoral dissertation]. California Institute of Technology. 